\title{
Substantiation of parameters of the energy- efficient water outflow scheme of underground mine using energy of hydro-flows
}

\author{
Marina Rylnikova ${ }^{1}$ and Egor Knyazkin ${ }^{1 *}$ \\ ${ }^{1}$ Federal State-Funded Research Establishment - Academician N. V. Melnikov Institute of \\ Comprehensive Exploitation of Mineral Resources, 111020, 4 Kryukovsky tupic, Moscow, Russia
}

\begin{abstract}
The purpose of the study is development of a structural scheme of mining-engineering system designed to ensure energy performance of the mine water outflow system. The article reveals the need for research aimed at improving the energy efficiency of the water outflow system by comparing the dynamics of electrical energy consumption and commercial output volume at the mining industry enterprises. A brief overview of the global experience in compensation for growth of electric energy consumption under more difficult mining conditions and mining depth due to the use of innovative technological solutions in the mine water outflow system is given. Scientific novelty of the research is the use of energy, which is bypassed to the underlying horizons of the underground mine, of technological and natural waters to improve efficiency of pumping units by using boost pressure and automated processes of cleaning hydraulic flows from sludge deposits, as well as to reduce the overall operational costs in the water outflow system. The energy-efficient scheme of mine water outflow system that increases energy efficiency of a mining enterprise is substantiated.
\end{abstract}

Keywords: mining engineering system, water outflow system, energy efficiency, hydraulic turbine, hydrocyclone, centrifugal pump, bypass borehole, liquid bypass height, useful power, mine pump.

\section{Introduction}

Reducing the specific energy intensity for the product is the basis for improving the competitiveness of domestic mining enterprises. Therefore, effective use of energy resources in the mine engineering system is extremely important at the current stage of economic development and consists in reducing the amount of energy spent on output of a commercial product unit.

The government of the Russian Federation also takes stimulating actions aimed at improving the energy efficiency of production facilities regulated by Federal law N 261-FZ "On energy saving and energy efficiency improvement and on amendments to certain legislative acts of the Russian Federation".

\footnotetext{
* Corresponding author: ea.knyazkin@gmail.com
} 
The need to reduce the energy component per output product unit in the mining industry is indicated by data from the official website of the Federal State Statistics Service (hereinafter — Rosstat) (Fig. 1) [1].

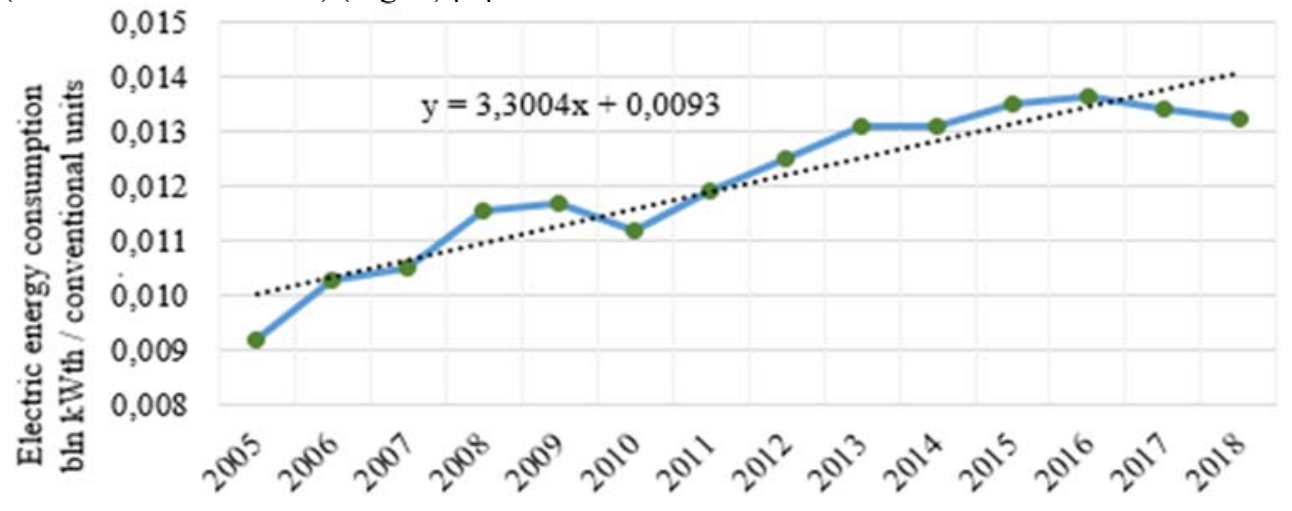

Fig. 1. Growth dynamics of electrical energy consumption for output of a commercial product unit in the mining industry

From the trend line equation in Fig. 1, plotted according to the Rosstat data, the growth trend of electric energy consumption for commercial product output in the mining industry over a 13-year period from 2005 to 2018 with a regression coefficient of 3.3004 is stable and equals to $42.9 \%$ or an average annual value of $3.3 \%$.

In the paper of D. R. Kaplunov and co-authors [2], the tendency of the energy intensity increase for mining products is primarily associated with the growing depth of mining operations. Factors determining the energy consumption growth in the structure of ore extraction cost are as follows:

- increased costs for the process of rock mass state control, which at great depths is usually associated with the need to use development systems with goaf stowing;

- increased costs for delivering and lifting the rock mass to the surface, water outflow and ventilation of the underground mine.

Reducing the energy intensity of these processes is possible by improving mechanization of the mining operations, as well as by finding energy-efficient technical and technological solutions that are particularly important at great depths.

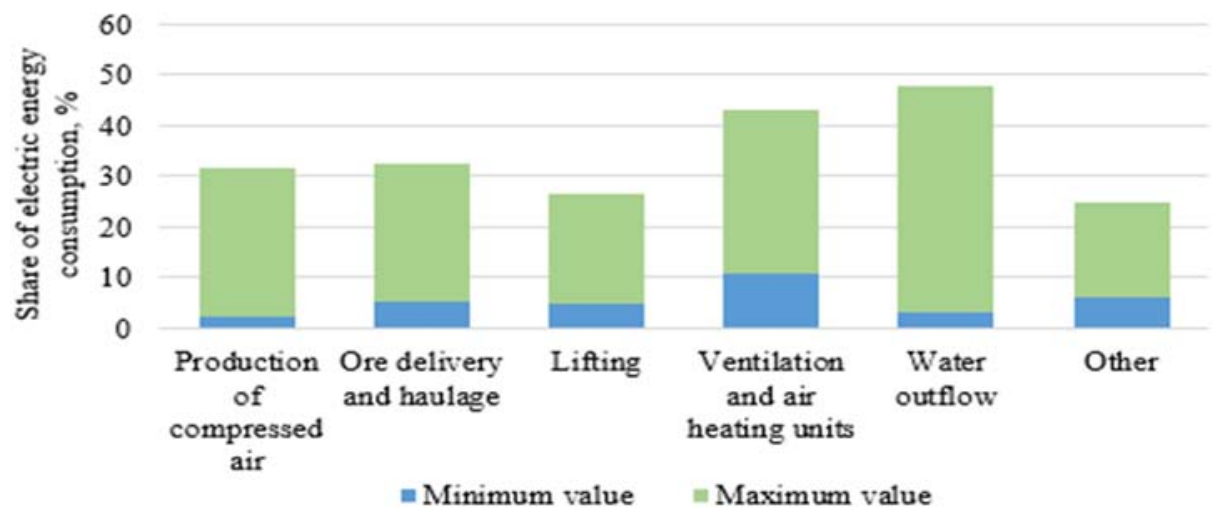

Fig. 2. Share of electric energy consumption by technological processes from the total electric energy consumption by the mine

Based on analysis and generalization of the research results by domestic and foreign specialists [3-7], the most energy-intensive technological processes for extraction of solid minerals were systematized and identified, as shown in Fig. 2, which are expressed as a 
percentage to the total power consumption of the mine in a representative sampling based on the analysis of 14 mining enterprises in Russia, near and far abroad.

From the histogram analysis (Fig. 2), it can be seen that the maximum electric consumption of the water outflow system is significantly higher compared to other technological processes, which determines the relevance of research for energy intensity reduction of the water outflow system

\section{Materials and methods}

Thus, in the paper [8] on the Lucky Friday mine, the possibility of compensation for some electricity spent on the mine water diversion process by including a low-power hydro turbine mounted on vertical bypass pipelines in the cooling system of deep workings into the mining-engineering system was considered (Fig. 3).

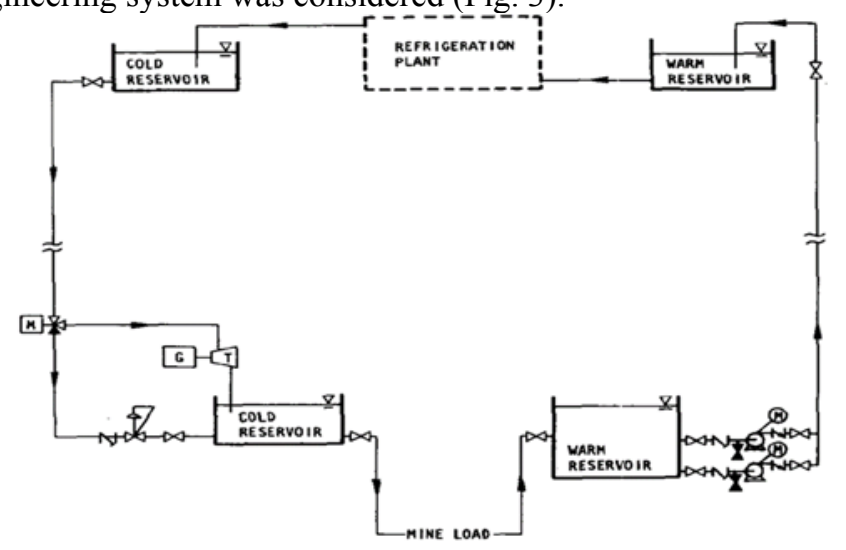

Fig. 3. Structure of the cooling system for deep workings of the Lucky Friday mine with an integrated hydraulic unit $(\mathrm{G}-\mathrm{T})$.

When using the energy of hydraulic flows freely moving under the action of gravity, various types of the hydro turbine "connections" were used in the design solutions at the Lucky Friday mine: with an electric generator - to compensate for part of the electric energy consumed when the liquid was lifted to the surface; with an air compressor - to create pumping of supplementary reservoir with compressed air used during mining operations; and with a hydraulic pump - to reduce mechanical load on the main water outflow pumping units.

It was revealed that combination of hydro turbine with an electric generator is preferred. In 1984, the designed unit was put into operation and was effectively used until the mine was closed. The considered option of compensating for the energy of free-flowing hydraulic flows undoubtedly has a sufficiently high potential, but requires high capital and operating costs. However, in practice, there are common examples of using centrifugal pumps operated in the hydro turbine mode, with decreased performance of the jet pressure.

Another promising solution for using the energy of bypassed liquid is to design a water outflow system with the possibility of using one of the main laws of hydrostatics - the law of communicating vessels. The proposed idea is applied in the invention [9], in which the pressure of the bypassed mine water is used as the initial boost for the mine centrifugal pump. At the same time, the traditional scheme of mine water outflow does not change (Fig.4). 


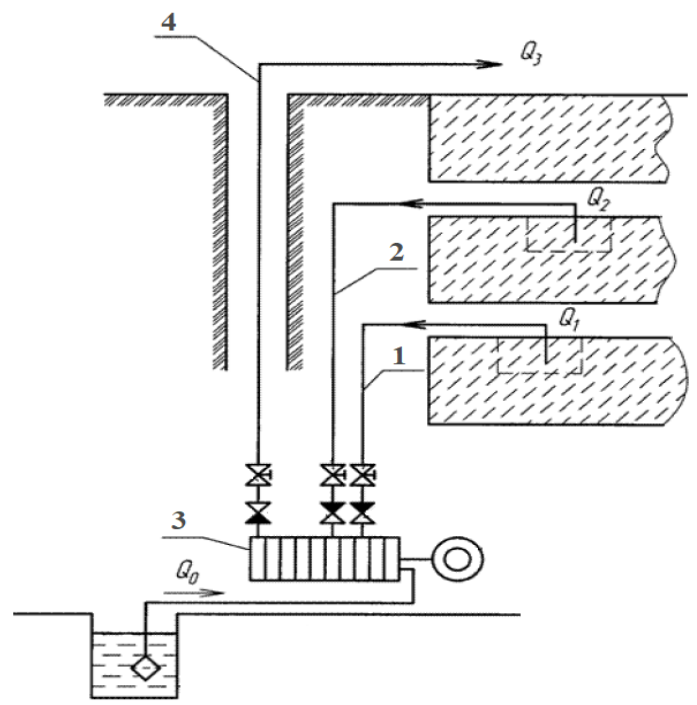

Fig. 4. Scheme of water outflow using the energy of the bypassed mine water; 1 and 2 - bypass pipelines, 3 - hydraulic installation, 4 - high-pressure discharge pipeline.

The scheme shown in Fig. 4 has two significant drawbacks:

- clarification of the bypassed mine water is carried out by settling in intermediate sumps, which requires additional capital investment for their construction and operation;

- there is no possibility of operational control over the system during periods of drastic changes of the mine water inflow in the dynamics of annual fluctuations due to the connection of bypass pipelines 1 and 2 with different-pressure sections of the centrifugal pump.

The idea of communicating vessels is used in the improved structural scheme of water outflow, devoid of the above disadvantages (Fig. 5).

A distinctive feature of the developed water outflow scheme (Fig.5) is the absence of water sumps 6 at intermediate horizons 1, 2 and 3, located at heights H1, H2 and H3 relative to the main outflow horizon 4 , since the function of wastewater treatment from sludge deposits is performed by separating solid phase particles in the rotating liquid flow using a desludging device - hydrocyclone 8 . For its effective operation, the specified input hydraulic pressure is ensures, which in this scheme is created by gravity force of the water column with a height $\mathrm{H} 4$, determined according to the technical characteristics of the hydrocyclone. The water column pressure is created in the cavity of the bypass boreholes 5 with the use of accumulating reservoir 7 and is maintained dynamically in the given range due to changes in the rotation speed of hydraulic pump 10.

The developed scheme ensures reduction of the water outflow stations within the deposit up to one located on the lower horizon 4. This approach is energy efficient due to the use of pressure in pipeline 9 as boost pressure for the pumping unit, thereby reducing the load on electric drive.

Water outflow horizon 4 is drained by the traditional way using a water sump 11 of a reduced size relative to the dimensions used in the classic schemes of mine water outflow due to reducing the water inflow from intermediate horizons. Flows Q1 - Q3 through pipeline 9 fall into the pump cavity where they combine with flow Q4 from pipeline 12 and are lifted to the surface through pipeline 13. 


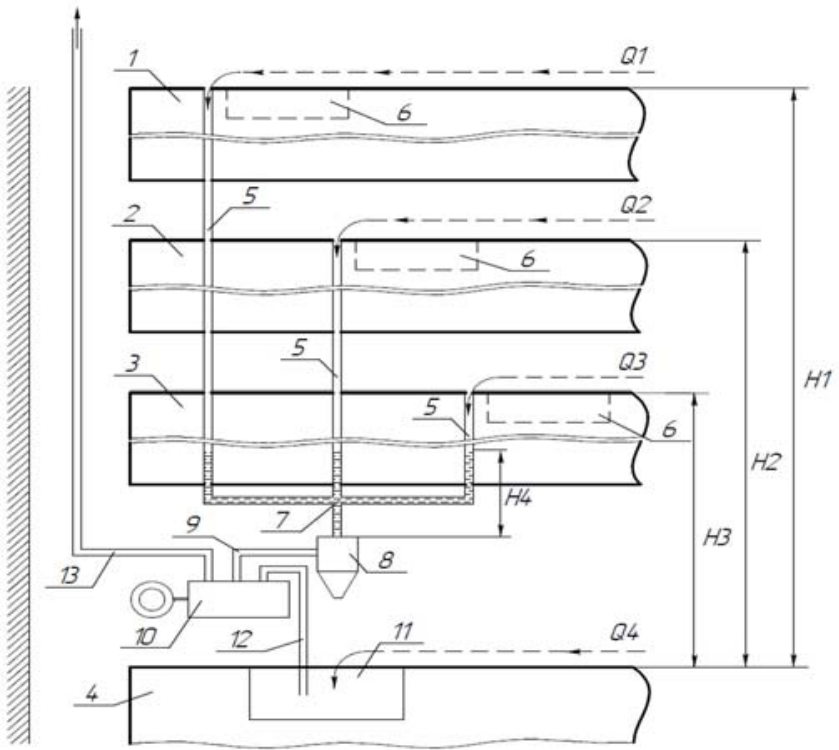

Fig. 5. Improved scheme of mine water outflow using the law of communicating vessels.

To confirm effectiveness of the improved water outflow scheme in accordance with the previously developed technique, a laboratory experiment was conducted, the scheme of which is shown in Figure 6.

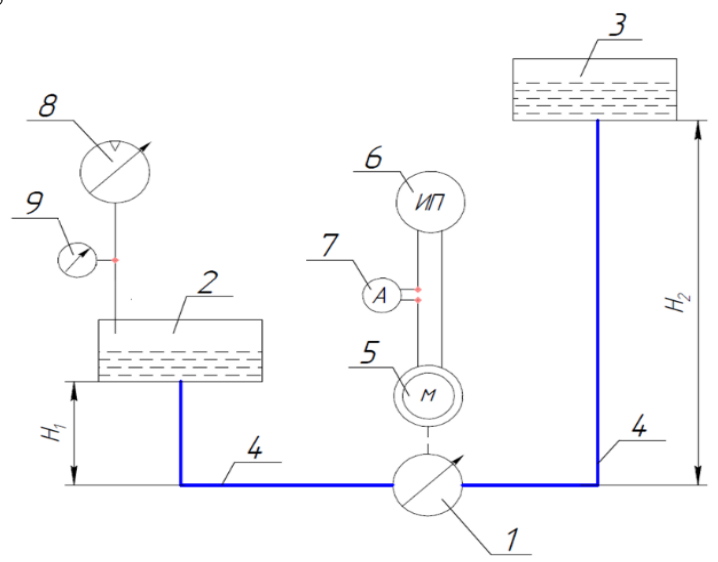

Fig. 6. Scheme of the laboratory experiment for substantiation of energy efficiency of the mine water outflow scheme.

The purpose of the experiment was to establish regularities of changing parameters of the developed water outflow scheme based on recording the changes in current consumption by electric drive 5 of hydraulic pump 1 connected to the power source 6 via ammeter 7, as a result of pressure water jet impact into the intake nozzle of pump 1 . The liquid is pumped from container 2 to the container 3, located at different heights $\mathrm{H} 1$ and $\mathrm{H} 2$ to ensure, respectively, the head and resistance of the liquid flow moving through pipeline 4. To create a large range of height change H1, air compressor 8 was connected to sealed container 2 via pressure regulator 9 .

The mine water outflow elements used in the laboratory experiment are shown in Figure 7. The low-power laboratory pump 1 in a combined moisture-proof case was used as an electric drive and hydraulic pump (Fig. 7 a), container 2 and pressure regulator 9 are shown in Figure $7 \mathrm{~b}$, current consumption of the electric pump was measured using a multimeter turned on in the ammeter mode (Fig.7 c). 
a)

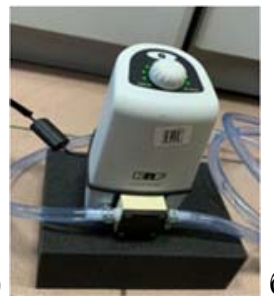

б)

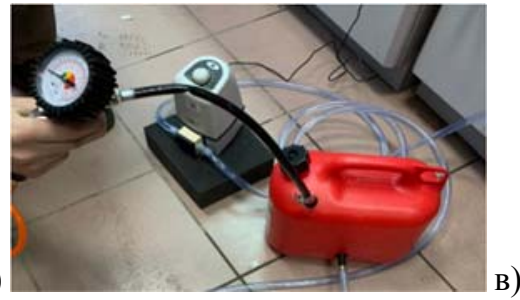

Fig. 7. Elements of the laboratory experiment scheme: low-flow laboratory pump in the united waterproof case (a), general presentation of the experiment part to be studied and adjusted in the form of the electric pump, sealed container with the liquid and pressure regulator (b), multimeter in the mode of the ammeter mounted in the power-supply circuit break of the electric pump (in).

\section{Results and discussion}

Results of the experiment confirmed the viability of the proposed water outflow scheme. Thus, when boost water pressure for the pump was increasing in vessel 2, current consumption of the electric drive was proportionally reducing and tending to be minimal as the pressure in container 2 was increasing up to the level of height H2 (Fig. 6).

These experimental results are explained by the influence of boost hydraulic pressure on the useful power of the pump $\mathrm{Nu}$ :

$$
\mathrm{Nu}=\mathrm{Q} \cdot \rho \cdot \mathrm{g} \cdot \mathrm{H},[\mathrm{W}]
$$

where $\mathrm{Q}$ - volume liquid flow, $\left[\mathrm{m}^{3} / \mathrm{s}\right] ; \mathrm{g}$ - acceleration of gravity $\left[\mathrm{m} / \mathrm{s}^{2}\right] ; \mathrm{p}$ - density of the pumped liquid, $\left[\mathrm{kg} / \mathrm{m}^{3}\right] ; \mathrm{H}$ - head of the water jet $[\mathrm{m}]$, which shall be created by the pump to ensure the given height of the liquid lifting.

The required head of the water jet in this case is determined from the difference between the head height $\mathrm{H} 2$ and the inlet boost height $\mathrm{H} 1$, thus reducing the overall useful power of the pump.

Then, the efficiency coefficient of the hydraulic pump $\eta$ is calculated based on the ratio of the useful power $\mathrm{Nu}$ to the rated power of the selected hydraulic unit:

$$
\eta=\mathrm{Nu} / \mathrm{N}
$$

where $\mathrm{N}$ - rated power of the pumping unit [W].

Thus, the use of the mine water bypass pressure makes it possible to "lower" the required head for the pumping unit, increasing its efficiency by $15-20 \%$ and, as a result, to reduce electrical energy consumption when locating the water outflow station on the lower horizon.

\section{Conclusions}

Summarizing the above, we can point out the following advantages of the developed water outflow scheme:

- cutting the operational costs of the system by reducing the number of pumping stations, as the scheme includes a single point of pumping mine water and provides increasing efficiency by partially compensating the required pump head with the boost pressure of the bypass hydraulic flows;

- reducing the labor costs for preparation and maintenance of intermediate water sumps due to automated treatment of waste water from sludge deposits using a hydrocyclone;

- increasing the degree of water discharge process automation, for example, by control of the hydraulic pump speed depending on the water column height that provides boost pressure for the hydrocyclone; 
- operational flexibility due to design optimization and location of bypass boreholes of the water outflow scheme in the deposits that differ significantly from each other in hydrogeological parameters.

The study was carried out with financial support of the Russian Foundation for Basic Research within the scientific project No. 19-35-90099

\section{References}

1. Online-resource: https://www.gks.ru/enterprise_industrial (2020)

2. D. R. Kaplunov, M. V. Rylnikova, V. V. Eks. Mining Informational and Analytical Bulletin (scientific and technical journal), 6, 5, (2014)

3. D. R. Kaplunov, M. V. Rylnikova. Gornyi Zhurnal (Engineering \& Mining Journal, 9,72, (2015)

4. L. F van der Zee, R. Pelzer and G. Bolt. International Conference on the Eleventh Industrial and Commercial Use of Energy (2014)

5. D. R. Kaplunov, V. A. Yukov. Mining Informational and Analytical Bulletin, 4, 5, (2016)

6. D. I. Bleiwas. US Geological Survey,108, (2011)

7. E. Jarvie-Eggart Michelle. Englewood, Colorado: Society for Mining, Metallurgy and Exploration, 804 (2015)

8. R. Torbin. IEEE TRANSACTIONS ON INDUSTRY APPLICATIONS, 5, 25 (1989)

9. V. V. Volkov, D. V. Volkov, Yu. P. Stashinov. Russian patent № 2454568, 18 (2012) 